\title{
Global miRNA expression of bone marrow mesenchymal stem/stromal cells derived from Fanconi anemia patients
}

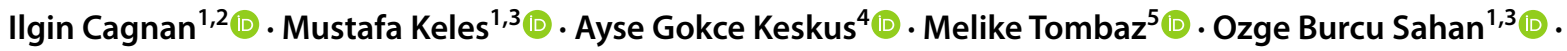 \\ Fatima Aerts-Kaya $^{1,3}$ (1) Duygu Uckan-Cetinkaya ${ }^{1,3,6}$ (1) $\cdot$ Ozlen Konu ${ }^{4,5}$ (D) $\cdot$ Aysen Gunel-Ozcan ${ }^{1,3}$ (1)
}

Received: 8 January 2021 / Accepted: 26 September 2021 / Published online: 18 November 2021

(c) The Author(s) under exclusive licence to Japan Human Cell Society 2021

\begin{abstract}
Fanconi anemia (FA) is a rare genetic disorder characterized by genomic instability, developmental defects, and bone marrow (BM) failure. Hematopoietic stem cells (HSCs) in BM interact with the mesenchymal stem/stromal cells (MSCs); and this partly sustains the tissue homeostasis. MicroRNAs (miRNAs) can play a critical role during these interactions possibly via paracrine mechanisms. This is the first study addressing the miRNA profile of FA BM-MSCs obtained before and after BM transplantation (preBMT and postBMT, respectively). Non-coding RNA expression profiling and quality control analyses were performed in Donors $(n=13)$, FA preBMT $(n=11)$, and FA postBMT $(n=6)$ BM-MSCs using GeneChip miRNA 2.0 Array. Six Donor-FA preBMT pairs were used to identify a differentially expressed miRNA expression signature containing 50 miRNAs, which exhibited a strong correlation with the signature obtained from unpaired samples. Five miRNAs (hsamiR-146a-5p, hsa-miR-148b-3p, hsa-miR-187-3p, hsa-miR-196b-5p, and hsa-miR-25-3p) significantly downregulated in both the paired and unpaired analyses were used to generate the BM-MSCs' miRNA-BM mononuclear mRNA networks upon integration of a public dataset (GSE16334; studying Donor versus FA samples). Functionally enriched KEGG pathways included cellular senescence, miRNAs, and pathways in cancer. Here, we showed that hsa-miR-146a-5p and hsa-miR-874-3p were rescued upon BMT ( $n=3$ triplets). The decrease in miR-146a-5p was also validated using RT-qPCR and emerged as a strong candidate as a modulator of BM mRNAs in FA patients.
\end{abstract}

Keywords Fanconi anemia $\cdot$ Bone marrow $\cdot$ Mesenchymal stem/stromal cells $\cdot$ miRNA $\cdot$ Non-coding RNAs

\section{Introduction}

MicroRNAs (miRNAs) are a class of short non-coding RNAs that degrade mRNAs or repress their translation [1]. During embryonic development and adulthood, miRNAs play key roles in the regulation of various biological and

Ilgin Cagnan, Mustafa Keles, Ayse Gokce Keskus and Melike Tombaz have equally contributed to this manuscript and are ordered according to the alphabetic list of their surnames.

Ozlen Konu

konu@fen.bilkent.edu.tr

$\triangle$ Aysen Gunel-Ozcan

aysen.ozcan@hacettepe.edu.tr

1 Department of Stem Cell Sciences, Graduate School of Health Sciences, Center for Stem Cell Research and Development, Hacettepe University, 06100 Sihhiye, Ankara, Turkey

2 Present Address: Department of Biological Sciences, Faculty of Arts and Sciences, Eastern Mediterranean University, 99628 Famagusta, North Cyprus, via Mersin-10, Turkey physiological processes, including hematopoiesis, immunity,

3 Center for Stem Cell Research and Development, PEDI-STEM, Hacettepe University, 06100 Sihhiye, Ankara, Turkey

4 Interdisciplinary Neuroscience Program, Bilkent University, Ankara, Turkey

5 Department of Molecular Biology and Genetics, Bilkent University, Ankara, Turkey

6 Department of Pediatrics, Division of Bone Marrow Transplantation Unit, Faculty of Medicine, Hacettepe University, Ankara, Turkey 
metabolism, apoptosis, and cell cycle, as well as in the differentiation and maintenance of tissue identity. Abnormalities in miRNA expression or regulation have been associated with numerous human disease states, including Fanconi anemia [2-5].

Fanconi anemia (FA) is a rare genetic disorder that can be caused by mutations in at least 22 different genes encoding proteins that play a role in the FANC/BRCA pathway $[6,7]$. FA proteins mediate the repair of interstrand crosslinks via homologous recombination [8], while FA is characterized by genomic instability, developmental defects, high cancer risk (i.e., acute myeloid leukemia and solid organ tumors), as well as bone marrow failure caused by myelodysplastic syndrome (MDS) and/or aplastic anemia (AA) [9]. Bone marrow transplantation (BMT) is currently the only available treatment for the hematopoietic defects seen in FA patients [10]. Bone marrow failure due to defective hematopoiesis is primarily caused by genetic and environmental alterations, but also abnormal interactions between the hematopoietic cells and bone marrow mesenchymal stem/stromal cells (BM-MSCs) [11].

BM-MSCs are multipotent stem cells and shown to differentiate into components (e.g., cells of the osteogenic lineage, pericytes, and endothelial cells) of the bone marrow microenvironment or niche, which are required for functional hematopoiesis $[12,13]$. A recent study suggests that an abnormal bone marrow niche plays a role in the hematopoietic defects seen in FA patients [14]. In $\mathrm{Fancc}^{-/}$; Fancg $^{-/}$double knockout mouse models, MSCs are suggested to have defective osteogenic differentiation capacity, as well as decreased hematopoietic supportive activity [14]. Therefore, MSCs regulate both hematopoietic stem cell (HSC) self-renewal and differentiation in part by paracrine action [15]. miRNAs secreted from bone marrow niche cells may alter hematopoietic cell function. A study investigating exosomal miRNAs in severe AA and MDS shows the importance of circulating miRNAs for the progression of bone marrow failure [16]. Additionally, exosomal miRNAs such as miR-125a-3p, miR-146a, miR-21-5p, miR-142-3p, miR-223-3p, and miR-126-3p derived from BM-MSCs are shown to either modulate inflammation or maturation/differentiation of hematopoietic cells such as $\mathrm{T}$ lymphocytes and dendritic cells [17-19]. The limited studies currently available on the role of miRNAs in FA pathogenesis mainly focus on miRNA expression in bone marrow mononuclear cells (BM-MNCs) [3-5].

MicroRNA (miRNA) expression can change by replicative senescence in BM-MSCs of healthy donors with or without chromosomal stability $[20,21]$. Therefore, we hypothesized that miRNA regulatory networks in FA BM-MSCs might be affected by the genomic instability or early senescence seen in these patients. In this respect, we aimed to comparatively identify the miRNA expression profile of in vitro expanded BM-MSCs derived from FA patients and donors to decode their relevance in FA pathology. To our knowledge, this is the first study that shows how miRNA expression profile changes in paired (i.e., related) FA and donor samples followed by comparisons made with unpaired, as well as postBMT samples. A recent report with the largest sibling cohort of FA up to date indicates the importance of genotypic and phenotypic data from FA siblings and shows a strong correlation between the progressions of hematological manifestations between affected siblings [22]. Therefore, comparing miRNA profiles between FA and their related-donor samples may further highlight the possible alterations in FA transcriptomes, relatively independent of the genotypic background. Additionally, our network analyses and RT-qPCR results helped to validate and prioritize hsa-miR146a-5p and revealed candidate target-miRNA interaction modules in BM-MSC interaction in FA. Our findings also implicated that bone marrow transplantation may restore the expression level of several miRNAs dysregulated in FA BM-MSCs.

\section{Materials and methods}

\section{Bone marrow-derived mesenchymal stem/stromal cells from Fanconi anemia patients and donors}

Cryopreserved bone marrow mononuclear cells (MNCs) from FA patients obtained before (pre) and following (post) BMT at Hacettepe University Pediatric BMT unit were used for isolation of BM-MSCs. The study was approved by the Local Ethical Committee (Number 14, 24/08/2009). Informed consent was obtained from all individual participants/their parents included in the study.

Fanconi anemia (FA) patients were diagnosed by clinical manifestations and diepoxybutane (DEB) test $(n=11$; designated as HUSCS-FA), as described previously [23, 24]. postBMT BM-MSCs were obtained for HUSCS-FA02, HUSCS-FA04, HUSCS-FA06, HUSCS-FA09a, HUSCSFA10, and HUSCS-FA11. Healthy donors $(n=13$; designated as HUSCS-D) served as controls. HUSCS-D02, -D03, -D04, -D05, -D06, and -D07 were donors of FA patients, coded as HUSCS-FA05, -FA06, -FA08, -FA09a, -FA09b, and -FA11, respectively. Details of isolation and culture of BM-MSCs were described previously [23, 25]. Osteogenic and adipogenic in vitro differentiation capacity and immunophenotyping of FA preBMT and Donor BM-MSCs were described previously [23, 24]. Representative images showing the differentiation potential and immunophenotypes of postBMT BM-MSCs were given in the Online Resource 1. All the characterization of BM-MSCs was performed in the third passage. 


\section{Total RNA isolation}

Total RNA, including small RNAs, was isolated from passage 3 BM-MSCs using miRNeasy Mini Kit (Qiagen, Hilden) and treated with RNase-free DNase Digestion (Qiagen) according to the manufacturer's instruction, as described previously. The quantity of RNA samples was measured using a NanoDrop ND-1000 (Thermo Fisher Scientific, USA) spectrometer. RNA quality was assessed using an Agilent 2100 Bioanalyzer (Agilent Technologies Ireland Ltd., Cork, Ireland). Total RNA samples had RIN (RNA integrity number) values ranging between 7.70 and 10.0 (Online Resource 2).

\section{RT-qPCR for differentially expressed miRNA confirmation}

Total RNAs extracted from Donor $(n=10)$ and FA preBMT $(n=7)$ BM-MSCs (Online Resource 2$)$ were used for confirmation by RT-qPCR for the lead miRNA, hsa-miR146a-5p. cDNAs were synthesized from $5 \mathrm{ng}$ RNA samples (final concentration $=1 \mathrm{ng} / \mu \mathrm{l}$ ) using miRCURY LNA ${ }^{\mathrm{TM}}$ Universal RT microRNA PCR Starter Kit (Exiqon). qPCR was performed by 1/80 dilution of cDNA, miRCury LNA Exiqon primers, and SybrGreen mix (Exiqon) using the LightCycler 480 II instrument (Roche, USA). PCR conditions were $95^{\circ} \mathrm{C}$ for $10 \mathrm{~min}$ followed by 45 cycles of $95{ }^{\circ} \mathrm{C}$ for $10 \mathrm{~s}$ and $60{ }^{\circ} \mathrm{C}$ for $1 \mathrm{~min}$. Reactions were studied in duplicates. $\mathrm{Cp}$ values of hsa-miR146-5p were normalized to internal control miRNA hsa-let-7a-5p [26]. Relative gene expression was obtained by $\log$ transformation of $2^{-\Delta \mathrm{Cp}}$, and GraphPad Prism (Version 8.4.2) was used for analysis.

\section{Non-coding RNA expression profiling}

Global non-coding RNA (ncRNA) expression was assessed in Donor $(n=13)$, FA preBMT $(n=11)$, and FA postBMT $(n=6)$ BM-MSC samples. Total RNA including small RNAs (100 ng) was used to label RNAs with biotin using the Genisphere FlashTag HSR kit (Genisphere, Hatfield, PA). Subsequently, the samples were loaded to GeneChip miRNA 2.0 Array (Applied Biosystems, Thermo Fisher Scientific). All experimental services were purchased from AY-KA LTD (Ankara, Turkey) and were carried out following the manufacturer's protocol. Affymetrix GeneChip Command Console (AGCC) was used to determine the intensity value for each probe cell and to create a '.CEL' file for each sample. The flowchart used for processing CEL files can be found in Fig. 1. Quality control analysis $(n=30)$ and outlier detection were performed using NUSE from the affyPLM package in R [27]. After further validation via multidimensional scaling analysis (MDS), all detected outliers were removed (Fig. 1). CEL files and expression values for all three analyses were uploaded to Gene Expression Omnibus (GEO Number GSE183227).

\section{Donor-preBMT paired sample analysis}

For paired samples, rma normalization and batch correction between Donor and FA preBMT pairs $(n=6)$ were done using Transcriptome Analysis Console (TAC@ 2019, Thermo Fisher Scientific, version 4.0.2.15) to remove genetic background-related differences as described in TAC user manual. Since downstream analyses were based on human mature miRNAs, all non-human miRNAs and other non-coding RNAs were removed. The principal component analysis (PCA) was employed for the visualization of the results [28]. Limma analysis after the batch correction was performed for identifying the differentially expressed miRNAs of FA samples paired with their donors (Fig. 1) [29]. In addition, overall average mature human miRNA expression values of Donor and FA preBMT groups were compared via Student's $t$ test to check for any global difference in the expression. FDR adjusted $p$ value $<0.05$ and $\operatorname{abs}(\log F C)>0.5$ were used as the thresholds for further analyses. ComplexHeatmap package was used for clustering and plotting the heatmap with default linkage (complete) and distance (Euclidian) metrics [30]. Heatmap was annotated with the clinical information described in the manuscript by Cagnan et al. [24].

\section{Validation set with samples without a pair}

Fanconi anemia (FA) or donor samples without a pair were normalized using rma from the oligo package [31], and the differential gene expression analysis was performed using the limma package (Fig. 1) [29]. A Pearson's correlation coefficient was calculated between the $\log \mathrm{FC}$ values from paired samples and those with no pair and reported on the plot along with the associated $p$ value. miRNAs that were significant in both the paired and unpaired analyses were used in miRNA-mRNA network analysis.

\section{Donor-preBMT-postBMT paired sample analysis}

The three paired donor-preBMT together with their respective postBMT transcriptomes were subjected to the rma normalization followed by batch correction within each triplet using TAC (version 4.0.2.15) (Fig. 1). Differential gene expression analysis was performed between matched donor-preBMT, donor-postBMT, and preBMT-postBMT contrasts using limma. miRNAs with rescued expression in postBMT (i.e., significantly downregulated between donor-preBMT and preBMT-postBMT and insignificant in donor-postBMT) were selected for the network analysis $(n=2)$. 
Fig. 1 A flowchart depiction of the processing pipeline for CEL files used in this study (TAC Transcriptome Analysis Console; () 2019, Thermo Fisher Scientific, version 4.0.2.15)

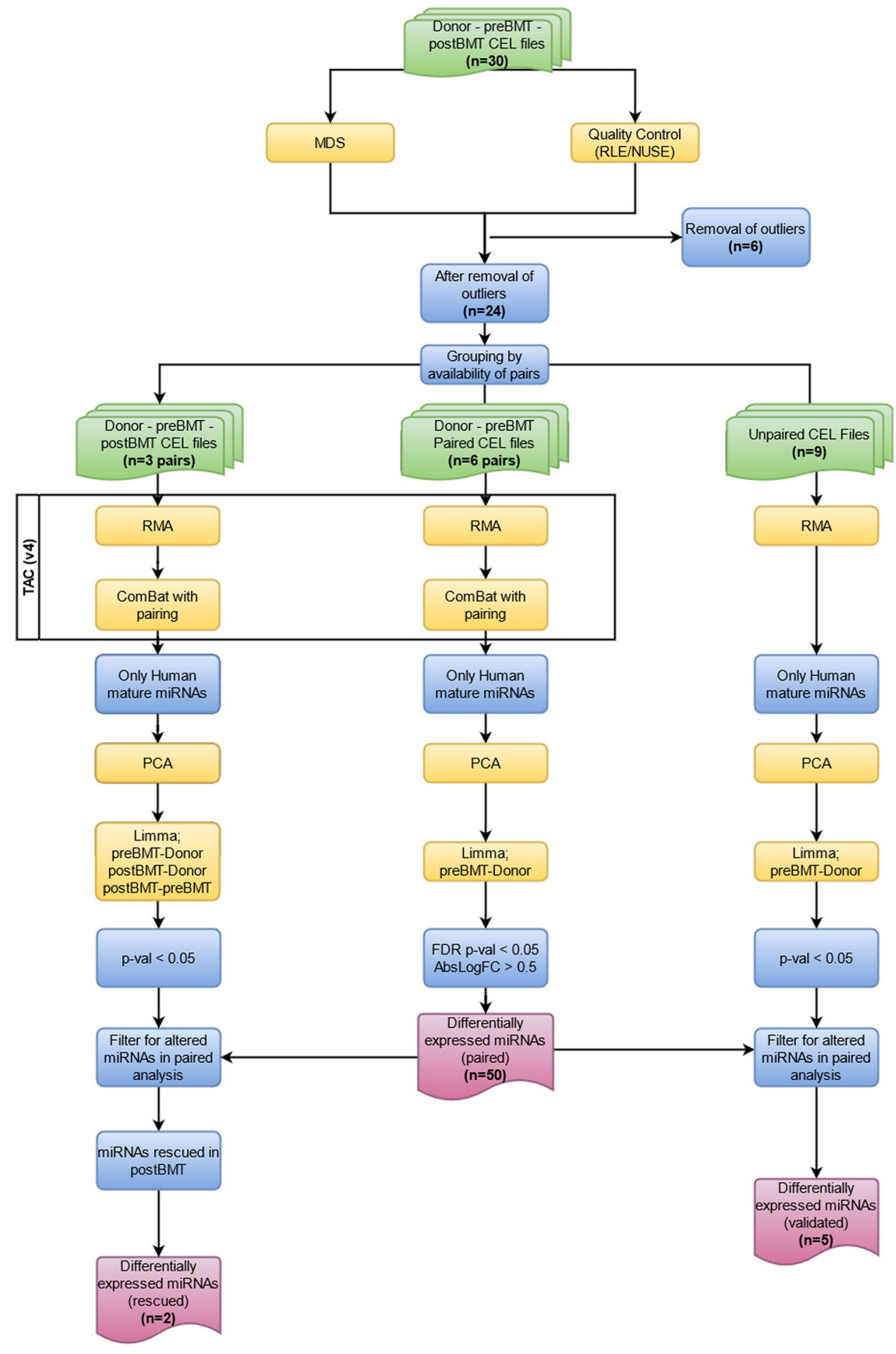

\section{Analysis of GSE16334 for filtering miRNA targets}

Raw CEL files of a public mRNA expression dataset for BM-MNCs of 11 Donors and 21 FA patients, GSE16334, were downloaded from the GEO database [32]. Differentially expressed mRNAs were determined using TAC (C version 4.0.2.15) software. Donors' and FA patients' expressions were compared using eBayes as the ANOVA method. mRNAs with significant adj. $p$ values (FDR $p$ value $<0.05$ ) were selected for downstream analyses. Significantly differentially expressed mRNA targets of the selected miRNAs used to generate miRNA-target networks. 


\section{Functional analysis of the pathways for predicted mRNA targets of the differentially expressed miRNAs in Fanconi anemia BM-MSCs}

Experimentally validated mRNA targets of the five miRNAs, which were significantly downregulated in both the paired and unpaired Donor-FA analyses (Fig. 1), were obtained from miRnet.ca (miRNet 2.0) [33], using miRTarBase (v8.0) and TarBase (v8.0). The targets were filtered down to differentially expressed genes found in GSE16334 with an FDR $p$ value $<0.05$. For differentially expressed genes, the probe set with the minimum $p$ value was selected for multiple probe sets belonging to the same gene symbol. Targets of miRNAs with an absolute $\log \mathrm{FC} \geq 0.5$ were used to generate the downregulated miRNA-upregulated mRNA networks in Cytoscape (v3.8.2) [34], in which logFC values of the genes were used to color the nodes, while the edge widths indicated the number of experimental evidence for the mRNA targets. Differentially expressed miRNA targets, which were targeted by at least two miRNAs, were subjected to functional enrichment analysis using the STRING database $[35,36]$.

For miRNAs with rescued expression in postBMT $(n=2)$, the functional enrichment analysis was performed for gene targets that were significantly upregulated in GSE16334 with an absolute $\log \mathrm{FC} \geq 0$ using the STRING database. To simplify the network, miRNA targets were filtered down to genes that were significantly upregulated in GSE16334 with a $\log F C \geq 0.5$ (FDR $p$ value $<0.05$ ). The downregulated miRNA-upregulated mRNA networks were generated in Cytoscape (v3.8.2).

\section{Results}

\section{Differentially expressed miRNAs between paired Fanconi anemia patients and donors}

Initially, the quality assessment of the raw microarray data was performed (Online Resource 3). Normalized Unscaled Standard Errors (NUSE) analysis revealed six possible outliers. After confirming the outliers through multidimensional scaling analysis (MDS), those samples were removed from further analysis (Online Resource 2; HUSCS-D13, HUSCSD16, HUSCS-FA02 preBMT, HUSCS-FA02 postBMT, HUSCS-FA04 postBMT, and HUSCS-FA10 postBMT).

The paired samples with FA preBMT and Donor $(n=6$; pairs) were initially subjected to batch effect correction between paired samples to remove genetic background associated differences (Fig. 1). The PCA analysis showed that Donor and FA preBMT samples were found in two separate clusters (Fig. 2a). Overall comparison of mature human miRNA expression values between Donor and FA preBMT samples showed no significant global change in the mean expression (average $\log 2$ expression: Donor $=3.893$ versus $\mathrm{FA}=3.856, p$ value $=0.7543$ ). After differential gene expression analysis with limma, we found significant differences in the expression levels of 50 miRNAs, i.e., 10 upregulated and 40 downregulated (Fig. 2b; Online Resource 4).

\section{miRNA-target network analysis of miRNAs differentially expressed in paired and unpaired samples}

Next, we analyzed the samples without pairs as a validation set complementing the paired analysis. We found a high positive correlation between logarithmically transformed fold change $(\log F C)$ values of the paired and those obtained from unpaired FA preBMT samples and independent donors, who are either donors of patients carrying other disease or donors without a paired FA patient (Fig. 2c; $r=0.55, p$ value $=2.904 \mathrm{e}-05$ ). Among the 50 differentially expressed miRNAs, five (hsa-miR-146a-5p, hsa-miR-148b-3p, hsamiR-187-3p, hsa-miR-196b-5p, and hsa-miR-25-3p) were significantly downregulated ( $p$ value $<0.05$ ) also in samples without a pair (Fig. 2c; Online Resource 5).

We further analyzed a public GSE16334 dataset containing mRNA expression data for 11 Donors and $21 \mathrm{FA}$ patients to examine the functions of miRNAs that were dysregulated in FA BM. We investigated the significantly modulated miRNA targets in FA BM-MNCs using the STRING database and Table 1 shows the number of miRNA targets obtained from miRNet.ca (Fig. 3a) and those found to be significantly dysregulated in the GSE16334 analysis (Fig. 3b). Venn diagrams [37] for five miRNA targets and significantly dysregulated targets showed that these miRNAs shared common targets. Common targets of all five miRNAs were TES and DICER1. DICERI was found to be downregulated in FA $\mathrm{BM}-\mathrm{MNCs}(\log \mathrm{FC}=-0.84$, FDR $p$ value $\leq 0.001)($ Fig. 3 ; Online Resource 6). We have also identified that targets of miR-146a-5p were significantly dysregulated (i.e., overexpressed) in BM of FA (Fisher's exact test; odds ratio $=2.20$, $p$ value $<0.0001$ ) along with those targets of three of the four remaining miRNAs (Online Resource 7).

The miRNA-target network constructed by Cytoscape pointed a large number of targets, one of which IFI27 was the most highly differentially expressed gene in the network $(\operatorname{logFC}=4.12$, adj. $p$ value $\leq 0.001)$, (Fig. 4). Upregulated genes targeted by at least two of the five downregulated miRNAs were used for the functional enrichment via STRING database; and our results revealed several KEGG pathways, including cellular senescence, microRNAs in cancer, and pathways in cancer, as significantly enriched (Table 2). WikiPathways analysis also provided several other enriched pathways for the miRNA-mRNA network, including TGF- $\beta$ signaling, and senescence and autophagy in cancer (Table 3). 
$\mathbf{a}$
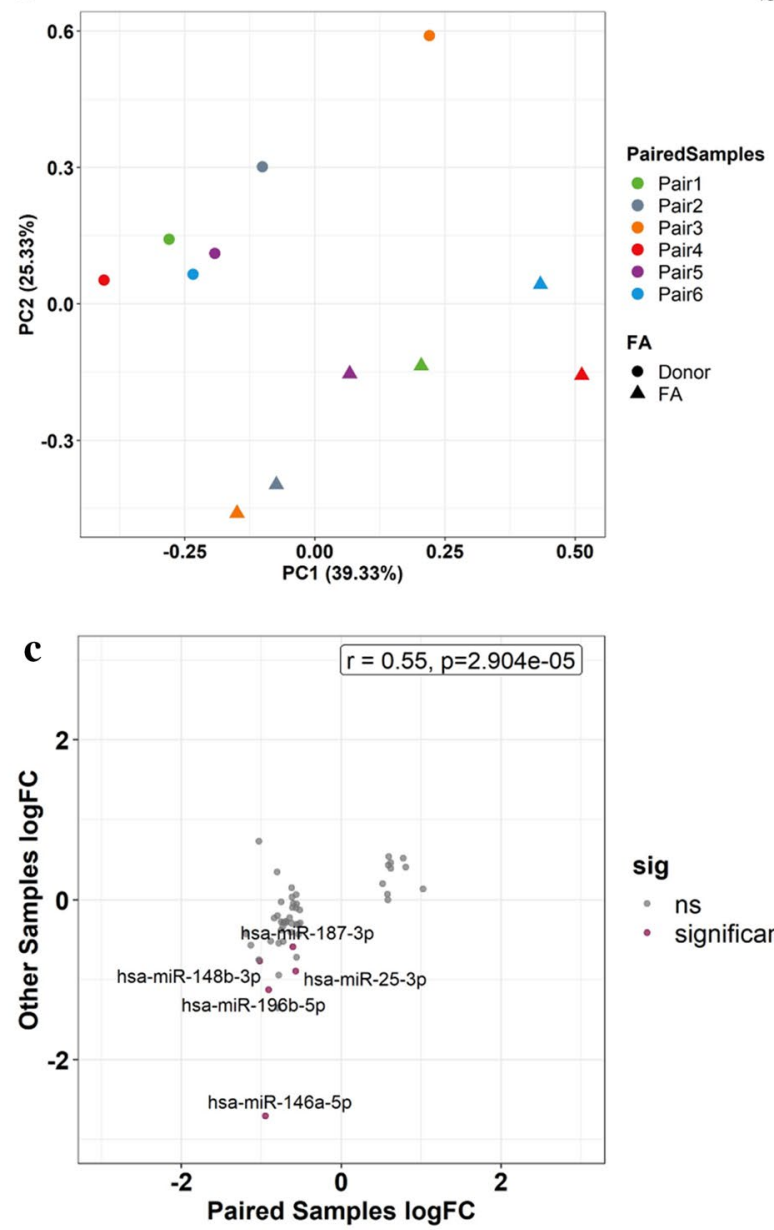

b

sig

ns

- significant

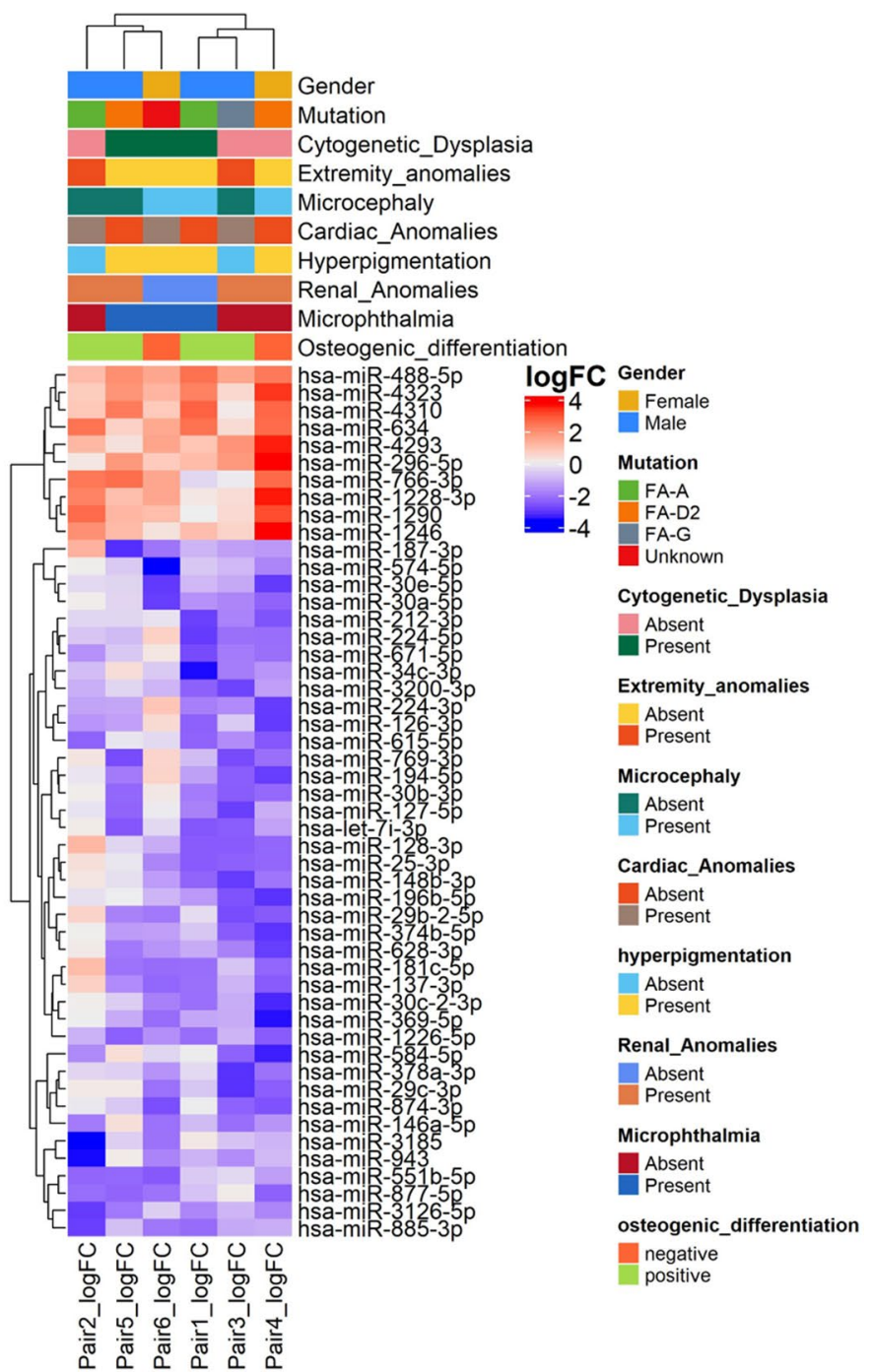

Samples on heatmap were annotated according to the patient characteristics and the color codes were shown on the legend. c Scatterplot of $\log \mathrm{FC}$ values of significantly altered miRNAs between the paired samples and unpaired preBMT-Donor comparisons. miRNAs altered significantly in both analyses were shown with red. Pearson's correlation coefficient and the associated $p$ values were reported on the plot
Table 1 Number of targets for miRNAs commonly downregulated in FA preBMT BM-MSCs based on both paired and unpaired analyses

\begin{tabular}{|c|c|c|c|c|c|}
\hline miRNA & Total \# of targets & $\begin{array}{l}\text { Targets in } \\
\text { GSE16334 }\end{array}$ & $\begin{array}{l}\text { Significant } \\
\text { targets }^{\mathrm{a}}\end{array}$ & $\begin{array}{l}\text { Upregulated } \\
\text { targets } \mathrm{s}^{\mathrm{b}}\end{array}$ & $\begin{array}{l}\text { Downregu- } \\
\text { lated targets }\end{array}$ \\
\hline hsa-miR-146a-5p & 4469 & 3178 & 2378 & 1385 & 993 \\
\hline hsa-miR-148b-3p & 1831 & 1469 & 1094 & 467 & 627 \\
\hline hsa-miR-25-3p & 1470 & 1174 & 904 & 331 & 573 \\
\hline hsa-miR-196b-5p & 842 & 673 & 502 & 180 & 322 \\
\hline hsa-miR-187-3p & 86 & 74 & 54 & 25 & 29 \\
\hline hsa-miR-874-3p & 350 & 278 & 203 & 91 & 112 \\
\hline
\end{tabular}

Significance of up/downregulation of mRNA targets are based on GSE16334

${ }^{\mathrm{a}} \mathrm{FDR}<0.05,{ }^{\mathrm{b}} \log \mathrm{FC} \geq 0,{ }^{\mathrm{c}} \log \mathrm{FC}<0$ 
Fig. 3 Venn diagrams for the common targets of five miRNAs. a Venn diagram for the total number of miRNA targets obtained from miRNet.ca. b Venn diagram for the miRNA targets that were significantly regulated in GSE16334 $($ FDR $<0.05)$
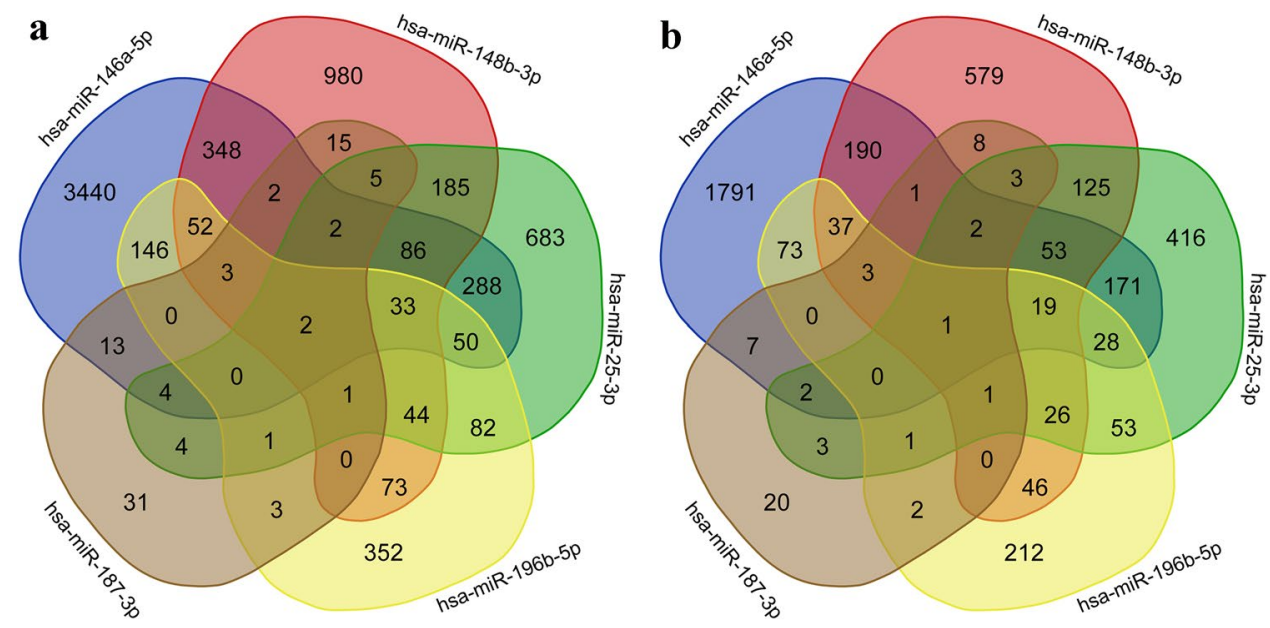

\section{Donor-preBMT-postBMT triplet analysis revealing the reversal of expression for the downregulated hsa-miR-146a after bone marrow transplantation}

Expression levels of Donor-preBMT samples were generally maintained even after postBMT, and multiple comparisons performed between preBMT-Donor, postBMT-Donor, and postBMT-preBMT (Fig. 1; Online Redource 2) revealed that preBMT samples were found closer to the cluster containing postBMT samples than the one containing donor (Fig. 5a, b). However, expressions of two miRNAs, i.e., hsa-miR-146a-5p and hsa-miR-874-3p, were found to be rescued after the allogeneic BMT (Fig. 5c). Further analysis on the expression levels of their mRNA targets (Table 1) indicated that some common targets of miR-146a-5p and miR-874-3p, such as GSPT1, FOXO3, and FOXN3, were upregulated in FA samples of GSE16334 (Online Resource 8). KEGG enrichment results for the upregulated targets of miR-146a-5p and miR-874-3p network included MAPK signaling pathway, pathways in cancer, PI3K signaling pathway, and FoxO signaling (Table 4).

The expression of hsa-miR-146a-5p was then confirmed by RT-qPCR using cDNAs obtained from total RNAs of Donor $(n=10)$ and FA preBMT $(n=7)$ BM-MSCs. Relative gene expression of hsa-miR-146a-5p, normalized to internal control hsa-miR-let-7a-5p, was significantly downregulated in $\mathrm{FA}$ preBMT samples $(\log \mathrm{FC}=-2.1$; average FA $-\Delta \mathrm{Cp}=-12.15$ versus Donor $-\Delta \mathrm{Cp}=-10.08, p$ value $=0.040$, Student's $t$ test, Fig. 6).

\section{Discussion}

In the present study, we performed non-coding RNA expression analysis in BM-MSCs from FA patients and healthy individuals using a paired analysis followed by validations performed with unpaired Donor and FA samples. Our analyses were further enriched by the addition of postBMT samples to identify miRNAs that could be reversed to their donor levels by BMT. In addition, we obtained the BM-MSCs miRNA-BM-MNC mRNA target network of FA-Donor samples, which were generated for the significant lead miRNAs. Among the significant miRNAs, we detected miR-146a-5p whose expression was significantly downregulated in both the paired and unpaired FA-Donor microarray comparisons and RT-qPCR; and rescued by BMT.

Herein, the miRNA-mRNA networks generated upon the incorporation of the public FA-donor MNC mRNA dataset makes the present study unique by identifying a potential paracrine cross-talk between MSCs and HSCs. We used a stringent analysis pipeline, which identified 50 miRNAs [with FDR < 0.05; abs $(\log F C)>0.5$ ] based on the expression analysis of six pairs of FA-Donors; and five (hsa-miR146a-5p, hsa-miR-148b-3p, hsa-miR-187-3p, hsa-miR196b-5p, and hsa-miR-25-3p) of these 50 were validated by unpaired analysis $(n=9)$. In addition, the expression of two (hsa-miR-146a-5p and hsa-miR-874-3p) of these were rescued after BMT based on our preBMT-postBMT-donor triplet analysis $(n=3)$.

Functional analysis of common targets of at least two of these five miRNAs helped identify enriched pathways in cellular senescence and cancer as well as TGF- $\beta$ signaling pathway. Additionally, functional analysis of the miRNA-mRNA network consisting of hsa-miR-146a-5p and hsa-miR-874-3p revealed that BM mRNAs targeted by the rescued miRNAs play roles in important pathways involved in MAPK, PI3KAkt, FoxO, and Ras signaling. Indeed, many of these signaling pathways or their components have already been shown to be dysregulated in FA cells or associated with FANC proteins [38-40]. In particular, the TGF- $\beta$ signaling pathway is persistently activated in FA, as well as the two main stress response signaling pathways, i.e., MAPK and NF-kB that 


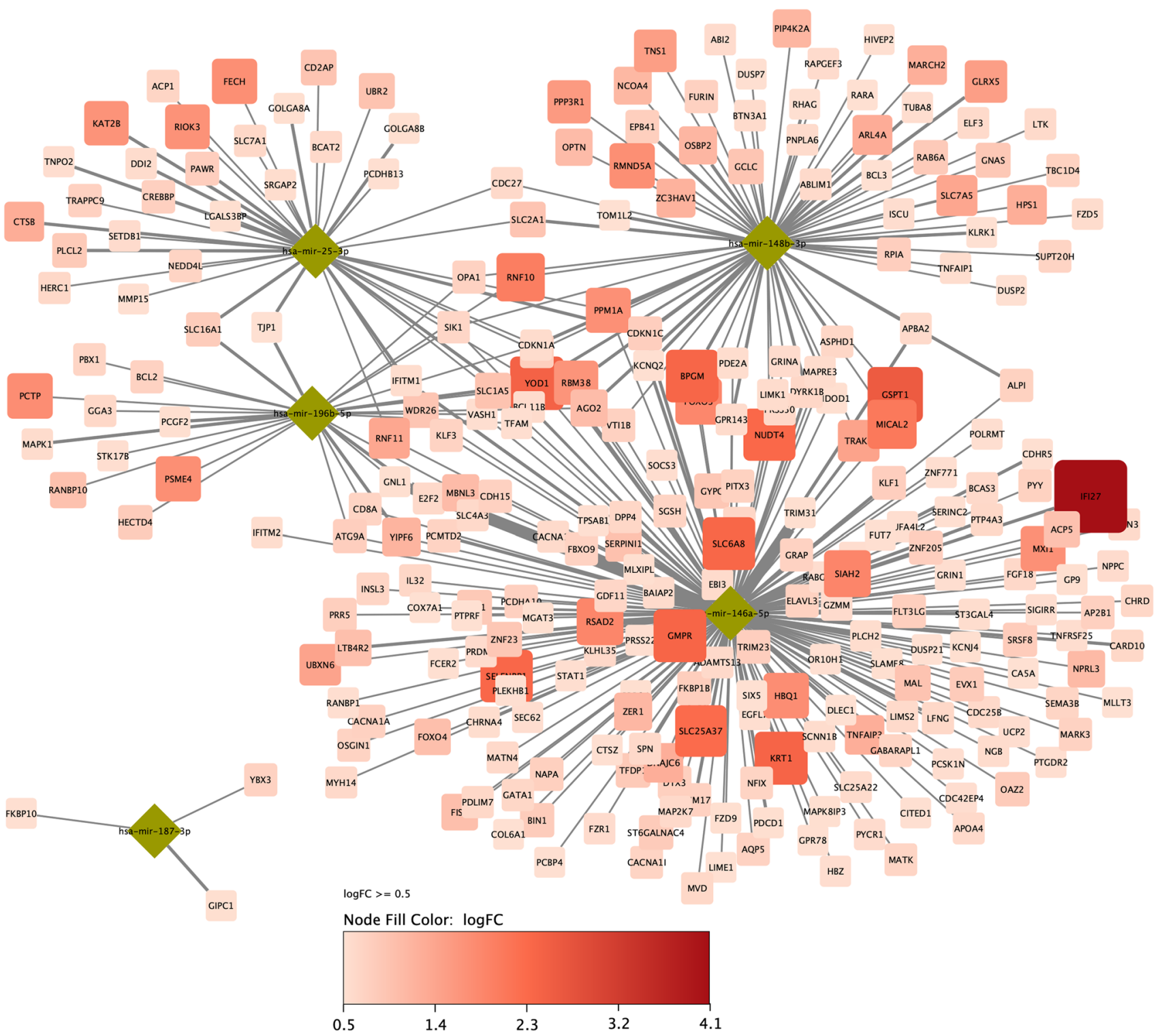

Fig. 4 miRNAs commonly downregulated in FA BM-MSCs in paired as well as unpaired analyses integrated with FA BM-MNCs mRNAs upregulated with a threshold of at least 0.5 (logFC). The miRNA-mRNA interaction data obtained from miRnet.ca were used in Cytoscape (v3.8.2); and coloring and size of mRNAs indicate the degree of upregulation. The edge width indicates the number of experimental evidences between the miRNA-mRNA pairs

MSC biology, such as regulation of cell cycle in Wharton's jelly umbilical cord stem cells or osteogenic differentiation of BM-MSCs and adipose-derived MSCs [44-46]. A recent study also has pinpointed the importance of MSC-secreted exosomal hsa-miR-25-3p as an anti-apoptotic agent in cardiomyocytes [47].

Fanconi anemia can lead to hematological disorders, such as AA and MDS. Giudice et al. extracted and profiled (via qPCR array) miRNAs from peripheral blood exosomes in MDS and severe aplastic anemia (SAA) patients [16]. Comparison of these results with our findings designated two common differentially expressed miRNAs (hsa-miR-25-3p 
Table 2 KEGG pathway enrichment results for genes that are upregulated and targeted by at least two miRNAs (false discovery rate $(\mathrm{FDR})<0.05$ and $\log \mathrm{FC} \geq 0.5)$

\begin{tabular}{llcl}
\hline \#Term ID & Term description & $\begin{array}{c}\text { Observed } \\
\text { gene count }\end{array}$ & FDR \\
\hline hsa05219 & Bladder cancer & 8 & 0.00056 \\
hsa04218 & Cellular senescence & 12 & 0.0036 \\
hsa05206 & MicroRNAs in cancer & 12 & 0.0044 \\
hsa01522 & Endocrine resistance & 9 & 0.0062 \\
hsa05200 & Pathways in cancer & 21 & 0.0221 \\
hsa04934 & Cushing syndrome & 10 & 0.0285 \\
hsa05131 & Shigellosis & 12 & 0.029 \\
hsa04371 & Apelin signaling pathway & 9 & 0.0297 \\
hsa05213 & Endometrial cancer & 6 & 0.0297 \\
hsa05205 & Proteoglycans in cancer & 11 & 0.0299 \\
hsa05202 & Transcriptional misregulation in & 10 & 0.0352 \\
& cancer & & \\
hsa05224 & Breast cancer & 9 & 0.0391 \\
hsa05223 & Non-small cell lung cancer & 6 & 0.0477 \\
hsa04390 & Hippo signaling pathway & 9 & 0.0478 \\
hsa05100 & Bacterial invasion of epithelial & 6 & 0.0478 \\
& cells & & \\
hsa05165 & Human papillomavirus infection & 14 & 0.0478 \\
hsa05218 & Melanoma & 6 & 0.0479 \\
\hline
\end{tabular}

and hsa-miR-30e-5p in SAA; hsa-miR-30e-5p in MDS) [16]. Further research on the role of these miRNAs may result in a better understanding of pathogenesis in SAA or MDS in FA patients. A miRNA we identified as downregulated in FA BM-MSCs, hsa-miR-181c (current miRBase ID: hsamiR-181c-5p) has also been found to be downregulated in both fresh peripheral blood MNCs and lymphoblastoid cell lines obtained from FA patients [4]; and yet, another differentially expressed miRNA from our study, hsa-miR-574-5p, has also been reported to be significantly downregulated in FA BM-MNC by Degan et al., who performed a microarray analysis on BM-MNCs derived from FA patients and healthy donors [3]. Subsequent research concerning these findings may further strengthen the role of miRNAs in the pathophysiology of FA.

Fanconi anemia (FA) cells are prone to DNA-damageinduced premature senescence and more importantly, FA cells including BM-MSCs have increased senescence activity when compared to controls [14, 48]. miRNAs are reported to regulate many cellular processes, including cellular senescence [49]. For example, Kundrotas and colleagues report downregulation of hsa-miR-187-3p, hsa-miR-224-5p, and hsa-miR-877-5p in the late passage (P6-P7) human BM-MSCs compared with early passage cells (P3-P4) [20]. Herein, we showed that expression of these three miRNAs also decreased in the third passage of FA BM-MSCs when compared to healthy individuals, thus supporting a possible role for these miRNAs during cellular senescence. Intriguingly, Pan et al. detected spontaneous transformation of BM and liver-derived MSCs with chromosomal number variations following long-term cultivation, and the expression of miR-187, miR-194, and miR-378 (current miRBase IDs: hsa-miR-187-3p, hsa-miR-194-5p, and hsa-miR-378a-3p, respectively) were upregulated in transformed MSCs compared to parental cells [21]. These results may indicate a change in the miRNA profile of genomically unstable MSCs to acquire the transformed state. Additionally, we showed decreased expression of hsa-miR-224-3p in FA preBMT BM-MSCs, although hsa-miR-224-3p expression was reported to increase as the passage of human umbilical cord or cord blood MSCs increased [50]. However, the difference between our results and the findings by Meng et al. may arise due to the use of MSCs from different sources.

Here, we also showed that hsa-miR146a-5p expression significantly decreased in FA preBMT BM-MSCs and its level was recovered to donor levels upon BMT. miR-146a (current miRBase ID: miR-146a-5p) has anti-inflammatory and tumor suppressor functions, while its mimics show
Table 3 WikiPathways enrichment results for genes that are upregulated and targeted by at least two miRNAs (false discovery rate $(\mathrm{FDR})<0.05$ and $\log \mathrm{FC} \geq 0.5)$

\begin{tabular}{llrl}
\hline \#Term ID & Term description & $\begin{array}{c}\text { Observed gene } \\
\text { count }\end{array}$ & FDR \\
\hline WP2828 & Bladder cancer & 7 & 0.019 \\
WP236 & Adipogenesis & 11 & 0.0193 \\
WP4533 & Transcription co-factors SKI and SKIL protein partners & 5 & 0.0193 \\
WP4659 & Gastrin signaling pathway & 10 & 0.0193 \\
WP2261 & Glioblastoma signaling pathways & 8 & 0.0392 \\
WP366 & TGF-beta signaling pathway & 10 & 0.0392 \\
WP615 & Senescence and autophagy in cancer & 9 & 0.0392 \\
WP4685 & Melanoma & 7 & 0.0427 \\
WP2813 & Mammary gland development pathway—embryonic develop- & 4 & 0.0444 \\
& ment (Stage 1 of 4) & & \\
WP4541 & Hippo-Merlin signaling dysregulation & 9 & 0.0472 \\
\hline
\end{tabular}




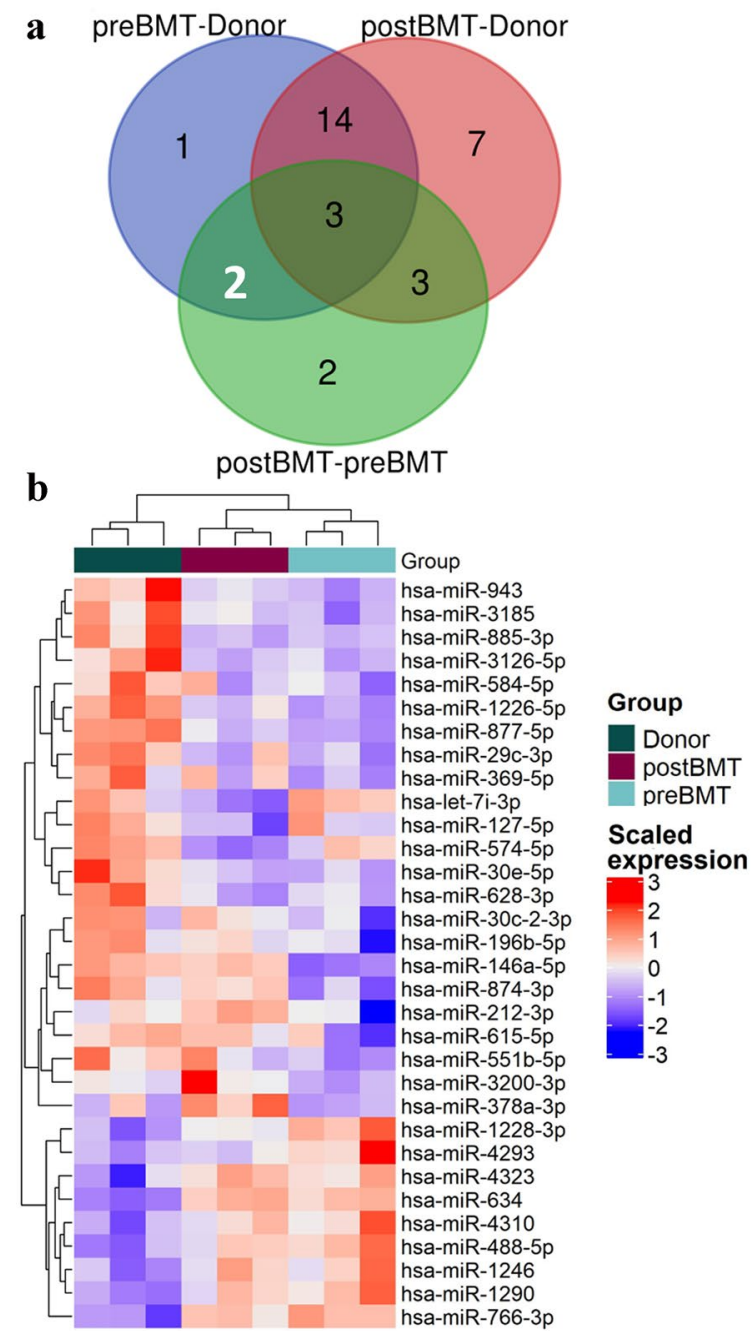

Fig. 5 Differentially expressed miRNAs in Donor, FA preBMT, and FA postBMT paired samples. a Venn diagram of significantly altered miRNAs in preBMT-Donor, postBMT-Donor, and postBMTpreBMT comparisons. b Heatmap representation of miRNAs altered

therapeutic potential also for inflammatory and myeloproliferative disorders [51]. miR-146a has been shown to inhibit NF-kB activation by targeting IRAK1 and TRAF6 [51]. In addition, NF-kB is induced in FA cells [52-54] and miR-146a can be stimulated by NF-kB subunit ReLA/ p65 overexpression, indicating a feedback loop [55, 56]. NF-kB activation is also involved in senescence-induced cytokine response [57] and can induce miR146a expression in cellular senescence. Moreover, excess miR-146a can impair DNA repair via targeting FANCM, a protein in FANC/BRCA pathway $[55,56]$. Interestingly, a study by Moussa Agha et al. showed that hsa-miR-146a was significantly upregulated in bone marrow fluid exosomes of AML patients compared to the healthy donors [58]. This suggests that overexpression of hsa-miR-146a-5p may lead

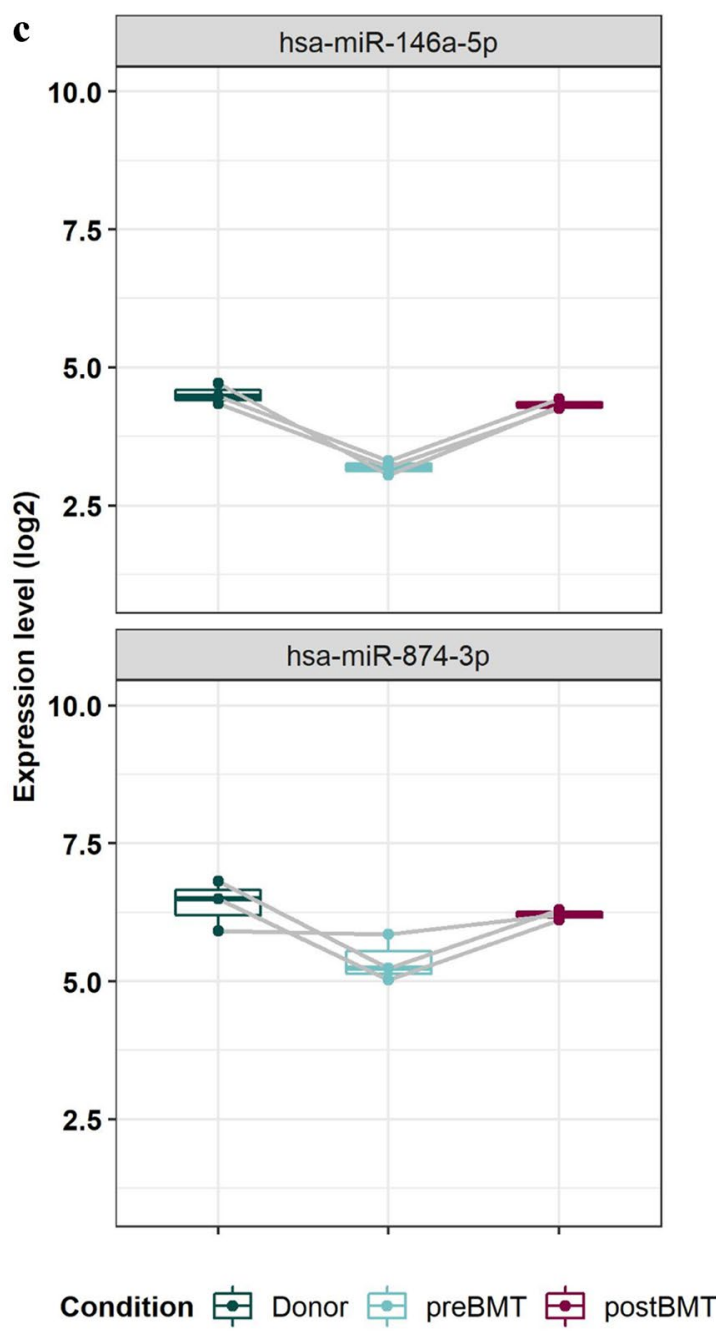

in at least one comparison shown in ' $\mathbf{a}$ ' Donor preBMT and postBMT status of samples were reported on the heatmap. $\mathbf{c}$ The boxplots obtained for the two miRNAs rescued, downregulated in preBMT, after the bone marrow transplant were labeled with white in part ' $a$ '

to uncontrolled proliferation of HSC progenitors, while its downregulation may cause aplasia.

Another important finding of our study was that a large proportion (74.8\%) of miR-146a-5p targets was modulated in the BM-MNC of FA patients. Among these targets, interferon-inducible gene 27 (IFI27), emerging as the most highly upregulated miR-146a target in FA BM-MNCs, is an oncogene highly expressed in myelofibrosis, and a well-known indicator of the burn-out phase of chronic inflammation leading to clonal evaluation and inefficient antitumor response [59]. All this current knowledge suggests that miR-146a dysregulation in FA BM-MSCs may be one of the underlying molecular mechanisms leading to the propensity of myeloid leukemia. Future studies using 
Table 4 Top 20 KEGG enrichment results for upregulated targets of hsa-miR-146a and hsa-miR-874-3p (false discovery rate $(\mathrm{FDR})<0.05$ and $\log \mathrm{FC} \geq 0$ )

\begin{tabular}{llll}
\hline \#Term ID & Term description & $\begin{array}{l}\text { Observed } \\
\text { gene } \\
\text { count }\end{array}$ & FDR \\
\hline hsa04010 & MAPK signaling pathway & 56 & $6.26 \mathrm{E}-07$ \\
hsa05224 & Breast cancer & 35 & $4.21 \mathrm{E}-06$ \\
hsa05200 & Pathways in cancer & 76 & $1.52 \mathrm{E}-05$ \\
hsa05205 & Proteoglycans in cancer & 40 & $1.52 \mathrm{E}-05$ \\
hsa04015 & Rap1 signaling pathway & 40 & $1.82 \mathrm{E}-05$ \\
hsa04151 & PI3K-Akt signaling pathway & 57 & $1.82 \mathrm{E}-05$ \\
hsa05226 & Gastric cancer & 32 & $2.41 \mathrm{E}-05$ \\
hsa01522 & Endocrine resistance & 24 & $8.86 \mathrm{E}-05$ \\
hsa04550 & Signaling pathways regulating & 30 & $8.86 \mathrm{E}-05$ \\
& pluripotency of stem cells & & \\
hsa05165 & Human papillomavirus infection & 51 & 0.00011 \\
hsa04014 & Ras signaling pathway & 39 & 0.00023 \\
hsa04068 & FoxO signaling pathway & 27 & 0.00023 \\
hsa05218 & Melanoma & 19 & 0.00039 \\
hsa04080 & Neuroactive ligand-receptor & 49 & 0.00048 \\
& interaction & & \\
hsa04360 & Axon guidance & 32 & 0.0005 \\
hsa04810 & Regulation of actin cytoskeleton & 35 & 0.0008 \\
hsa05215 & Prostate cancer & 21 & 0.0012 \\
hsa04510 & Focal adhesion & 33 & 0.0013 \\
hsa05219 & Bladder cancer & 13 & 0.0013 \\
hsa05223 & Non-small cell lung cancer & 17 & 0.0013 \\
\hline & & & \\
\hline
\end{tabular}

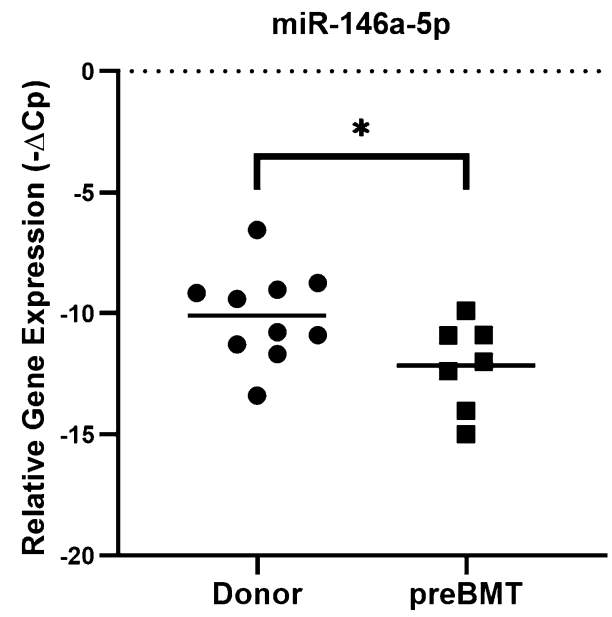

Fig. 6 Expression analysis of hsa-miR146a-5p by RT-qPCR in donor and FA preBMT BM-MSCs

miR-146a mimics are needed to examine the therapeutic role of this miRNA in improving the function of FA cells.

The TGF- $\beta$ signaling pathway has been identified as a major factor involved in the development of bone marrow failure in FA; and inhibition of the pathway rescues HSCs from genotoxicity via elevating homologous recombination while decreasing non-homologous end-joining repair [43]. A study in umbilical vein endothelial cells shows that miR-146a downregulates TGF- $\beta 1$ and miR-146a inhibitors upregulate TGF- $\beta 1$, as well as downstream proteins including phosphorylation of SMAD2 and PAI-1 [60]. Five of the downregulated miRNAs in our study, miR-146a-5p, miR-196b-3p, miR-148b-3p, and miR-25-3p (by paired and unpaired analysis) and miR-874-3p (only by paired analysis), target the TGF- $\beta$ pathway. Of these, the expression of miR146a-5p and miR-874-3p increased to the levels of healthy donors by BMT. Dysregulated TGF- $\beta$ signaling by impaired miRNAs seems to be the Achilles' heel of bone marrow in FA. Although upregulation of each of $T G F B 1, T G F B 2$, and SMAD6 was lower than 0.5-fold $(\log F C)$ in FA BM-MNCs, there were ten other significantly upregulated genes, with higher than 0.5 -fold changes $(\operatorname{logFC}$; Table 3$)$, involved in the TGF- $\beta$ signaling pathway; these genes were $C C N D 1$, TRAP1, THBS1, RBL2, SIK1, PPM1A, SP1, SKI, ITGB1, and $C D K N 1 A$ (GSE16334; [32]), and their upregulation is in favor of the aforementioned argument.

Moreover, the network analysis of downregulated targets has revealed a significant downregulation of DICERI, which is the key enzyme involved in dsRNA processing of siRNA and miRNA, as one of the common targets of the significantly downregulated five miRNAs (hsa-miR146a-5p, hsa-miR-196b-5p, hsa-miR-148b-3p, hsa-miR25-3p, and hsa-miR-187-3p) in FA BM-MSCs. Due to the downregulation of these miRNAs in FA BM-MSCs, one might expect upregulation of DICER1 in BM cells. Instead and as evidently, an overall increase of mature miRNAs in MSCs should be observed after DICER1 upregulation. However, there was no such bias for upregulated miRNAs in FA. Meanwhile, DICER1 in BM-MNCs was significantly downregulated with 1.79-fold change in FA (GSE16334). Accordingly, future studies should investigate the amount of change in DICER 1 in other components of the BM, especially the MSCs. However, there could still be multiple explanations for our finding. One possibility is that downregulation of expression in these miRNAs in BM-MSCs may not have a paracrine effect on HSC mRNAs or may exert a more complex regulation at the protein or post-transcriptional levels in the MNCs that affect the cross-talk of targets with the dilution of miRNAs [61], and warrants further study. Our study implicates several miRNAs downregulated in BM-MSCs as commonly targeting DICERI gene in support of roles of DICER in FA-related phenotypes. For example, Dicer1 knock-out mice osteoprogenitor (a component of bone marrow niche) cells display a MDS and AML-like phenotype [62], while DICER1 has also been shown to be downregulated in the BM-MSCs of MDS and AML patients [63]. On the other hand, it is upregulated in AML patient's HSCs as 
well as leukemia cell lines, and silencing of DICER1 suppresses leukemia cell proliferation and induces apoptosis [64]. Overall, this suggests that tightly controlled optimal levels of DICER1 in both HSCs and MSCs may be required for maintaining bone marrow homeostasis.

Intriguingly, in our study, hsa-miR-874-3p expression was downregulated in FA BM-MSCs and significantly reversed to donor levels following the BMT. miR-874-3p is shown to target and decrease the expression of $\mathrm{C}-\mathrm{X}-\mathrm{C}$ motif chemokine ligand 12 (CXCL12) in HEK293T and N2A cell lines [65]. CXCL12, also named as the stromal cellderived factor (SDF-1), is an essential chemoattractant for HSCs with a critical role in their localization to BM niche; and the cross-talk of CXCL12 with TGF- $\beta$ controls HSCs to stay in quiescence or switch to cycling state via PI3K/ Akt pathway downstream effectors FOXO3 and mTOR [66, 67]. hsa-miR-874-3p downregulation may lead to a possible upregulation of CXCL12 in FA BM-MSCs, which remains to be validated. Higher CXCL12 levels in turn may provide the feedback loop to protect HSC pool to rescue from aplasia or perhaps a cause leading to myelodysplasia. Previous research shows that CXCL12 is indeed upregulated in MDS BM-MSCs and proposed to be related to the hypercellularity of bone marrow seen in MDS patients [68]. Other studies also report the role of miR-874-3p in regulating the osteogenic differentiation of BM stromal cells as well as periodontal ligament stromal cells $[69,70]$, thus further showing the involvement of miR-874-3p in MSC biology. Future studies should focus on deciphering the role of miR-874-3p role in this feedback.

In conclusion, here, we compared the global miRNA expression in BM-MSCs derived from FA patients and those from donors, and showed potential interactions with mRNAs from BM-MNCs via generation of a novel paracrine miRNA-mRNA network in FA. Our data implicate that differences in miRNA expression may have impact on in vivo function of BM-MSCs. However, to establish a direct cause-effect relationship between the expression and functional contributions of miRNAs in FA BM-MSCs and its paracrine environment, further in vitro and in vivo studies are required.

Supplementary Information The online version contains supplementary material available at https://doi.org/10.1007/s13577-021-00626-9.

Acknowledgements This study was supported by The Scientific and Technological Research Council of Turkey (TUBITAK; project no: 110S021) in conjunction with EU COST Action BM0805 designated as 'HOX and TALE transcription factors in Development and Disease'.

Author contribution AGO designed the study and supervised the experiments. OK supervised the bioinformatics analysis. AGO, OK, IC, and MK drafted the introduction and discussion. IC, MK, AGK, MT, OBS, and FAK performed the experiments and/or data analysis. IC, MK, AGK, and MT drafted methods and results. DUC provided the bone marrow samples. All the authors approved the final version of manuscript.

Funding This work was supported by The Scientific and Technological Research Council of Turkey (TUBITAK; project no: 110S021) in conjunction with EU COST Action BM0805 designated as 'HOX and TALE transcription factors in Development and Disease'.

\section{Declarations}

Conflict of interest The authors have no relevant financial or non-financial interests to disclose.

Ethics approval This study was performed in line with the principles of the Declaration of Helsinki. This study was approved by the Local Ethical Committee (Number 14, 24/08/2009).

Informed consent Informed consent was obtained from all individual participants/their parents included in the study.

\section{References}

1. Bushati N, Cohen SM. microRNA functions. Annu Rev Cell Dev Biol. 2007;23:175-205. https://doi.org/10.1146/annurev.cellbio. 23.090506.123406.

2. Li Y, Kowdley KV. MicroRNAs in common human diseases. Genom Proteom Bioinform. 2012;10(5):246-53. https://doi.org/ 10.1016/j.gpb.2012.07.005.

3. Degan P, Cappelli E, Longobardi M, Pulliero A, Cuccarolo P, Dufour C, et al. A global MicroRNA profile in Fanconi anemia: a pilot study. Metab Syndr Relat Disord. 2019;17(1):53-9. https:// doi.org/10.1089/met.2018.0085.

4. Rio P, Agirre X, Garate L, Banos R, Alvarez L, San Jose-Eneriz E, et al. Down-regulated expression of hsa-miR-181c in Fanconi anemia patients: implications in TNFalpha regulation and proliferation of hematopoietic progenitor cells. Blood. 2012;119(13):30429. https://doi.org/10.1182/blood-2011-01-331017.

5. Suresh B, Kumar AM, Jeong HS, Cho YH, Ramakrishna S, Kim KS. Regulation of Fanconi anemia protein FANCD2 monoubiquitination by miR-302. Biochem Biophys Res Commun. 2015;466(2):180-5. https://doi.org/10.1016/j.bbrc.2015.08.127.

6. Knies K, Inano S, Ramirez MJ, Ishiai M, Surralles J, Takata M, et al. Biallelic mutations in the ubiquitin ligase RFWD3 cause Fanconi anemia. J Clin Invest. 2017;127(8):3013-27. https://doi. org/10.1172/JCI92069.

7. Mamrak NE, Shimamura A, Howlett NG. Recent discoveries in the molecular pathogenesis of the inherited bone marrow failure syndrome Fanconi anemia. Blood Rev. 2017;31(3):93-9. https:// doi.org/10.1016/j.blre.2016.10.002.

8. D'Andrea AD, Grompe M. The Fanconi anaemia/BRCA pathway. Nat Rev Cancer. 2003;3(1):23-34. https://doi.org/10.1038/nrc970.

9. Kee Y, D'Andrea AD. Molecular pathogenesis and clinical management of Fanconi anemia. J Clin Invest. 2012;122(11):3799_ 806. https://doi.org/10.1172/JCI58321.

10. Wagner JE, Eapen M, MacMillan ML, Harris RE, Pasquini R, Boulad F, et al. Unrelated donor bone marrow transplantation for the treatment of Fanconi anemia. Blood. 2007;109(5):2256-62. https://doi.org/10.1182/blood-2006-07-036657.

11. Kastrinaki MC, Pavlaki K, Batsali AK, Kouvidi E, Mavroudi I, Pontikoglou C, et al. Mesenchymal stem cells in immunemediated bone marrow failure syndromes. Clin Dev Immunol. 2013;2013: 265608. https://doi.org/10.1155/2013/265608. 
12. Morrison SJ, Scadden DT. The bone marrow niche for haematopoietic stem cells. Nature. 2014;505(7483):327-34. https://doi. org/10.1038/nature12984.

13. Muguruma Y, Yahata T, Miyatake H, Sato T, Uno T, Itoh J, et al. Reconstitution of the functional human hematopoietic microenvironment derived from human mesenchymal stem cells in the murine bone marrow compartment. Blood. 2006;107(5):1878-87. https://doi.org/10.1182/blood-2005-06-2211.

14. Zhou Y, He Y, Xing W, Zhang P, Shi H, Chen S, et al. An abnormal bone marrow microenvironment contributes to hematopoietic dysfunction in Fanconi anemia. Haematologica. 2017;102(6):1017-27. https://doi.org/10.3324/haematol.2016. 158717.

15. Chotinantakul K, Leeanansaksiri W. Hematopoietic stem cell development, niches, and signaling pathways. Bone Marrow Res. 2012;2012: 270425. https://doi.org/10.1155/2012/270425.

16. Giudice V, Banaszak LG, Gutierrez-Rodrigues F, Kajigaya S, Panjwani R, Ibanez M, et al. Circulating exosomal microRNAs in acquired aplastic anemia and myelodysplastic syndromes. Haematologica. 2018;103(7):1150-9. https://doi.org/10.3324/haema tol.2017.182824

17. Fujii S, Miura Y, Fujishiro A, Shindo T, Shimazu Y, Hirai H, et al. Graft-versus-host disease amelioration by human bone marrow mesenchymal stromal/stem cell-derived extracellular vesicles is associated with peripheral preservation of naive $\mathrm{T}$ cell populations. Stem Cells. 2018;36(3):434-45. https://doi.org/10.1002/ stem. 2759.

18. Reis M, Mavin E, Nicholson L, Green K, Dickinson AM, Wang XN. Mesenchymal stromal cell-derived extracellular vesicles attenuate dendritic cell maturation and function. Front Immunol. 2018;9:2538. https://doi.org/10.3389/fimmu.2018.02538.

19. Wu H, Fan H, Shou Z, Xu M, Chen Q, Ai C, et al. Extracellular vesicles containing miR-146a attenuate experimental colitis by targeting TRAF6 and IRAK1. Int Immunopharmacol. 2019;68:204-12. https://doi.org/10.1016/j.intimp.2018.12.043.

20. Kundrotas G, Gasperskaja E, Slapsyte G, Gudleviciene Z, Krasko J, Stumbryte A, et al. Identity, proliferation capacity, genomic stability and novel senescence markers of mesenchymal stem cells isolated from low volume of human bone marrow. Oncotarget. 2016;7(10):10788-802. https://doi.org/10.18632/oncotarget.7456.

21. Pan Q, Fouraschen SM, de Ruiter PE, Dinjens WN, Kwekkeboom J, Tilanus HW, et al. Detection of spontaneous tumorigenic transformation during culture expansion of human mesenchymal stromal cells. Exp Biol Med (Maywood). 2014;239(1):105-15. https://doi.org/10.1177/1535370213506802.

22. Jung M, Mehta PA, Jiang CS, Rosti RO, Usleaman G, Correa da Rosa JM, et al. Comparison of the clinical phenotype and haematological course of siblings with Fanconi anaemia. Br J Haematol. 2021;193(5):971-5. https://doi.org/10.1111/bjh.17061.

23. Cagnan I. HOX and TALE transcription factors in Fanconi anemia bonemarrow mesenchymal stem cells: gene expression and protein interactions. Ankara: Hacettepe University; 2018.

24. Cagnan I, Gunel-Ozcan A, Aerts-Kaya F, Ameziane N, Kuskonmaz B, Dorsman J, et al. Bone marrow mesenchymal stem cells carrying FANCD2 mutation differ from the other Fanconi anemia complementation groups in terms of TGF-beta1 production. Stem Cell Rev Rep. 2018;14(3):425-37. https://doi.org/10. 1007/s12015-017-9794-5.

25. Cagnan I, Aerts-Kaya F, Çetinkaya FD, Özcan A. Stably expressed reference genes during differentiation of bone marrow-derived mesenchymal stromal cells. Turk J Biol. 2017;41:88-97. https:// doi.org/10.3906/biy-1511-93.

26. Coste E, Rouleux-Bonnin F. The crucial choice of reference genes: identification of miR-191-5p for normalization of miRNAs expression in bone marrow mesenchymal stromal cell and
HS27a/HS5 cell lines. Sci Rep. 2020;10(1):17728. https://doi.org/ 10.1038/s41598-020-74685-7.

27. Brettschneider J, Collin F, Bolstad BM, Speed TP. Quality assessment for short oligonucleotide microarray data. Technometrics. 2008;50(3):241-64. https://doi.org/10.1198/004017008000000 334.

28. Pearson FRSK. LIII. On lines and planes of closest fit to systems of points in space. Lond Edinb Dublin Philos Mag J Sci. 1901;2(11):559-72. https://doi.org/10.1080/14786440109462720.

29. Ritchie ME, Phipson B, Wu D, Hu Y, Law CW, Shi W, et al. limma powers differential expression analyses for RNA-sequencing and microarray studies. Nucleic Acids Res. 2015;43(7): e47. https://doi.org/10.1093/nar/gkv007.

30. Gu Z, Eils R, Schlesner M. Complex heatmaps reveal patterns and correlations in multidimensional genomic data. Bioinformatics. 2016;32(18):2847-9. https://doi.org/10.1093/bioinformatics/ btw313.

31. Carvalho BS, Irizarry RA. A framework for oligonucleotide microarray preprocessing. Bioinformatics. 2010;26(19):2363-7. https://doi.org/10.1093/bioinformatics/btq431.

32. Vanderwerf SM, Svahn J, Olson S, Rathbun RK, Harrington C, Yates J, et al. TLR8-dependent TNF-(alpha) overexpression in Fanconi anemia group C cells. Blood. 2009;114(26):5290-8. https://doi.org/10.1182/blood-2009-05-222414.

33. Chang L, Zhou G, Soufan O, Xia J. miRNet 2.0: network-based visual analytics for miRNA functional analysis and systems biology. Nucleic Acids Res. 2020;48(W1):W244-51. https://doi.org/ 10.1093/nar/gkaa467.

34. Shannon P, Markiel A, Ozier O, Baliga NS, Wang JT, Ramage D, et al. Cytoscape: a software environment for integrated models of biomolecular interaction networks. Genome Res. 2003;13(11):2498-504. https://doi.org/10.1101/gr.1239303.

35. Szklarczyk D, Gable AL, Lyon D, Junge A, Wyder S, HuertaCepas J, et al. STRING v11: protein-protein association networks with increased coverage, supporting functional discovery in genome-wide experimental datasets. Nucleic Acids Res. 2019;47(D1):D607-13. https://doi.org/10.1093/nar/gky1131.

36. Szklarczyk D, Gable AL, Nastou KC, Lyon D, Kirsch R, Pyysalo S, et al. The STRING database in 2021: customizable proteinprotein networks, and functional characterization of user-uploaded gene/measurement sets. Nucleic Acids Res. 2021;49(D1):D60512. https://doi.org/10.1093/nar/gkaa1074.

37. Bioinformatics \& Evolutionary Genomics. 2021. http://bioinforma tics.psb.ugent.be/webtools/Venn/. Accessed 30 Aug 2021.

38. Li J, Du W, Maynard S, Andreassen PR, Pang Q. Oxidative stress-specific interaction between FANCD2 and FOXO3a. Blood. 2010;115(8):1545-8. https://doi.org/10.1182/ blood-2009-07-234385.

39. Oppezzo A, Bourseguin J, Renaud E, Pawlikowska P, Rosselli F. Microphthalmia transcription factor expression contributes to bone marrow failure in Fanconi anemia. J Clin Invest. 2020;130(3):1377-91. https://doi.org/10.1172/JCI131540.

40. Zhang X, Lu X, Akhter S, Georgescu MM, Legerski RJ. FANCI is a negative regulator of Akt activation. Cell Cycle. 2016;15(8):1134-43. https://doi.org/10.1080/15384101.2016. 1158375.

41. Briot D, Mace-Aime G, Subra F, Rosselli F. Aberrant activation of stress-response pathways leads to TNF-alpha oversecretion in Fanconi anemia. Blood. 2008;111(4):1913-23. https://doi.org/10. 1182/blood-2007-07-099218.

42. Svahn J, Lanza T, Rathbun K, Bagby G, Ravera S, Corsolini F, et al. p38 Mitogen-activated protein kinase inhibition enhances in vitro erythropoiesis of Fanconi anemia, complementation group A-deficient bone marrow cells. Exp Hematol. 2015;43(4):295-9. https://doi.org/10.1016/j.exphem.2014.11.010. 
43. Zhang H, Kozono DE, O'Connor KW, Vidal-Cardenas S, Rousseau A, Hamilton A, et al. TGF-beta inhibition rescues hematopoietic stem cell defects and bone marrow failure in Fanconi anemia. Cell Stem Cell. 2016;18(5):668-81. https://doi.org/10. 1016/j.stem.2016.03.002.

44. Gu H, Xu J, Huang Z, Wu L, Zhou K, Zhang Y, et al. Identification and differential expression of microRNAs in 1, 25-dihydroxyvitamin D3-induced osteogenic differentiation of human adipose-derived mesenchymal stem cells. Am J Transl Res. 2017;9(11):4856-71.

45. Han X, Yang H, Liu H, Zhang C, Cao Y, Fan Z, et al. miR196b-5p inhibits proliferation of Wharton's jelly umbilical cord stem cells. FEBS Open Bio. 2021;11(1):278-88. https://doi.org/ 10.1002/2211-5463.13043.

46. Mollazadeh S, Fazly Bazzaz BS, Neshati V, de Vries AAF, Naderi-Meshkin H, Mojarad M, et al. Overexpression of MicroRNA-148b-3p stimulates osteogenesis of human bone marrowderived mesenchymal stem cells: the role of MicroRNA-148b-3p in osteogenesis. BMC Med Genet. 2019;20(1):117. https://doi. org/10.1186/s12881-019-0854-3.

47. Peng Y, Zhao JL, Peng ZY, Xu WF, Yu GL. Exosomal miR-25-3p from mesenchymal stem cells alleviates myocardial infarction by targeting pro-apoptotic proteins and EZH2. Cell Death Dis. 2020;11(5):317. https://doi.org/10.1038/s41419-020-2545-6.

48. Zhang X, Sejas DP, Qiu Y, Williams DA, Pang Q. Inflammatory ROS promote and cooperate with the Fanconi anemia mutation for hematopoietic senescence. J Cell Sci. 2007;120(Pt 9):1572-83. https://doi.org/10.1242/jcs.003152.

49. Suh N. MicroRNA controls of cellular senescence. BMB Rep. 2018;51(10):493-9.

50. Meng X, Xue M, Xu P, Hu F, Sun B, Xiao Z. MicroRNA profiling analysis revealed different cellular senescence mechanisms in human mesenchymal stem cells derived from different origin. Genomics. 2017;109(3-4):147-57. https://doi.org/10.1016/j. ygeno.2017.02.003.

51. Su YL, Wang X, Mann M, Adamus TP, Wang D, Moreira DF, et al. Myeloid cell-targeted miR-146a mimic inhibits NF-kappaBdriven inflammation and leukemia progression in vivo. Blood. 2020;135(3):167-80. https://doi.org/10.1182/blood.2019002045.

52. Epanchintsev A, Shyamsunder P, Verma RS, Lyakhovich A. IL-6, IL-8, MMP-2, MMP-9 are overexpressed in Fanconi anemia cells through a NF-kappaB/TNF-alpha dependent mechanism. Mol Carcinog. 2015;54(12):1686-99. https://doi.org/10.1002/mc. 22240.

53. Kontou M, Adelfalk C, Hirsch-Kauffmann M, Schweiger M. Suboptimal action of NF-kappaB in Fanconi anemia cells results from low levels of thioredoxin. Biol Chem. 2003;384(10-11):1501-7. https://doi.org/10.1515/BC.2003.166.

54. Li Y, Li X, Cole A, McLaughlin S, Du W. Icariin improves Fanconi anemia hematopoietic stem cell function through SIRT6mediated NF-kappa B inhibition. Cell Cycle. 2018;17(3):367-76. https://doi.org/10.1080/15384101.2018.1426413.

55. Sundaravinayagam D, Kim HR, Wu T, Kim HH, Lee HS, Jun S, et al. miR146a-mediated targeting of FANCM during inflammation compromises genome integrity. Oncotarget. 2016;7(29):45976-94. https://doi.org/10.18632/oncotarget.10275.

56. Sundaravinayagam D, Kim HR, Wu T, Kim HH, Lee HS, Jun S, et al. Correction: miR146a-mediated targeting of FANCM during inflammation compromises genome integrity. Oncotarget. 2020;11(21):2024-5. https://doi.org/10.18632/oncotarget.27481.

57. Jing H, Lee S. NF-kappaB in cellular senescence and cancer treatment. Mol Cells. 2014;37(3):189-95. https://doi.org/10.14348/ molcells.2014.2353.
58. Moussa Agha D, Rouas R, Najar M, Bouhtit F, Naamane N, Fayyad-Kazan $\mathrm{H}$, et al. Identification of acute myeloid leukemia bone marrow circulating MicroRNAs. Int J Mol Sci. 2020. https:// doi.org/10.3390/ijms21197065.

59. Skov V, Larsen TS, Thomassen M, Riley CH, Jensen MK, Bjerrum OW, et al. Whole-blood transcriptional profiling of interferon-inducible genes identifies highly upregulated IFI27 in primary myelofibrosis. Eur J Haematol. 2011;87(1):54-60. https:// doi.org/10.1111/j.1600-0609.2011.01618.x.

60. Li Y, Zhu H, Wei X, Li H, Yu Z, Zhang H, et al. LPS induces HUVEC angiogenesis in vitro through miR-146a-mediated TGFbeta1 inhibition. Am J Transl Res. 2017;9(2):591-600.

61. Arvey A, Larsson E, Sander C, Leslie CS, Marks DS. Target mRNA abundance dilutes microRNA and siRNA activity. Mol Syst Biol. 2010;6:363. https://doi.org/10.1038/msb.2010.24.

62. Raaijmakers MH, Mukherjee S, Guo S, Zhang S, Kobayashi T, Schoonmaker JA, et al. Bone progenitor dysfunction induces myelodysplasia and secondary leukaemia. Nature. 2010;464(7290):852-7. https://doi.org/10.1038/nature08851.

63. Ozdogan H, Gur Dedeoglu B, Oztemur Islakoglu Y, Aydos A, Kose S, Atalay A, et al. DICER1 gene and miRNA dysregulation in mesenchymal stem cells of patients with myelodysplastic syndrome and acute myeloblastic leukemia. Leuk Res. 2017;63:6271. https://doi.org/10.1016/j.leukres.2017.10.006.

64. Bai Y, Qiu GR, Zhou F, Gong LY, Gao F, Sun KL. Overexpression of DICER1 induced by the upregulation of GATA1 contributes to the proliferation and apoptosis of leukemia cells. Int J Oncol. 2013;42(4):1317-24. https://doi.org/10.3892/ijo.2013.1831.

65. Xie K, Cai Y, Yang P, Du F, Wu K. Upregulating microRNA874-3p inhibits CXCL12 expression to promote angiogenesis and suppress inflammatory response in ischemic stroke. Am J Physiol Cell Physiol. 2020;319(3):C579-88. https://doi.org/10.1152/ajpce 11.00001 .2020

66. Chabanon A, Desterke C, Rodenburger E, Clay D, Guerton B, Boutin L, et al. A cross-talk between stromal cell-derived factor- 1 and transforming growth factor-beta controls the quiescence/ cycling switch of CD34(+) progenitors through FoxO3 and mammalian target of rapamycin. Stem Cells. 2008;26(12):3150-61. https://doi.org/10.1634/stemcells.2008-0219.

67. Sugiyama T, Kohara H, Noda M, Nagasawa T. Maintenance of the hematopoietic stem cell pool by CXCL12-CXCR4 chemokine signaling in bone marrow stromal cell niches. Immunity. 2006;25(6):977-88. https://doi.org/10.1016/j.immuni.2006.10. 016.

68. Flores-Figueroa E, Varma S, Montgomery K, Greenberg PL, Gratzinger D. Distinctive contact between CD34+ hematopoietic progenitors and CXCL12+CD271+ mesenchymal stromal cells in benign and myelodysplastic bone marrow. Lab Invest. 2012;92(9):1330-41. https://doi.org/10.1038/labinvest.2012.93.

69. Huang Y, Han Y, Guo R, Liu H, Li X, Jia L, et al. Long non-coding RNA FER1L4 promotes osteogenic differentiation of human periodontal ligament stromal cells via miR-874-3p and vascular endothelial growth factor A. Stem Cell Res Ther. 2020;11(1):5. https://doi.org/10.1186/s13287-019-1519-z.

70. Kushwaha P, Khedgikar V, Sharma D, Yuen T, Gautam J, Ahmad N, et al. MicroRNA 874-3p exerts skeletal anabolic effects epigenetically during weaning by suppressing Hdac1 expression. J Biol Chem. 2016;291(8):3959-66. https://doi.org/10.1074/jbc.M115. 687152 .

Publisher's Note Springer Nature remains neutral with regard to jurisdictional claims in published maps and institutional affiliations. 